\title{
Altered gene expression profiles in the hippocampus and prefrontal cortex of type 2 diabetic rats
}

\author{
Omar Abdul-Rahman ${ }^{1}$, Maria Sasvari-Szekely ${ }^{1}$, Agota Ver ${ }^{1}$, Klara Rosta ${ }^{1}$, Bernadett K Szasz ${ }^{2}$, Eva Kereszturi ${ }^{1}$ and \\ Gergely Keszler ${ }^{1 *}$
}

\begin{abstract}
Background: There has been an increasing body of epidemiologic and biochemical evidence implying the role of cerebral insulin resistance in Alzheimer-type dementia. For a better understanding of the insulin effect on the central nervous system, we performed microarray-based global gene expression profiling in the hippocampus, striatum and prefrontal cortex of streptozotocin-induced and spontaneously diabetic Goto-Kakizaki rats as model animals for type 1 and type 2 diabetes, respectively.

Results: Following pathway analysis and validation of gene lists by real-time polymerase chain reaction, 30 genes from the hippocampus, such as the inhibitory neuropeptide galanin, synuclein gamma and uncoupling protein 2, and 22 genes from the prefrontal cortex, e.g. galanin receptor 2, protein kinase C gamma and epsilon, ABCA1 (ATPBinding Cassette A1), CD47 (Cluster of Differentiation 47) and the RET (Rearranged During Transfection) protooncogene, were found to exhibit altered expression levels in type 2 diabetic model animals in comparison to non-diabetic control animals. These gene lists proved to be partly overlapping and encompassed genes related to neurotransmission, lipid metabolism, neuronal development, insulin secretion, oxidative damage and DNA repair. On the other hand, no significant alterations were found in the transcriptomes of the corpus striatum in the same animals. Changes in the cerebral gene expression profiles seemed to be specific for the type 2 diabetic model, as no such alterations were found in streptozotocin-treated animals.
\end{abstract}

Conclusions: According to our knowledge this is the first characterization of the whole-genome expression changes of specific brain regions in a diabetic model. Our findings shed light on the complex role of insulin signaling in fine-tuning brain functions, and provide further experimental evidence in support of the recently elaborated theory of type 3 diabetes.

\section{Background}

Diabetes mellitus is a chronic and heterogenous metabolic disorder affecting millions of patients worldwide. Type 1 diabetes is characterized by absolute insulin deficiency due to viral or autoimmune destruction of pancreatic beta cells, while the major feature of the more common type 2 variant is obesity-linked impairment of intracellular insulin signaling [1-3]. Apart from its wellknown effect on blood sugar levels, insulin is known to regulate the growth, differentiation and metabolism of its

\footnotetext{
* Correspondence: gergely.keszler@eok.sote.hu

'Department of Medical Chemistry, Molecular Biology and

Pathobiochemistry, Semmelweis University, Budapest, Hungary

Full list of author information is available at the end of the article
}

target cells at multiple levels [1]. Insulin signaling pathways have been shown to converge on and modulate the transcription of a plethora of genes [2]. In light of this, it is not surprising that gene expression microarrays revealed dramatic alterations in global gene expression profiles of several organs such as skeletal muscles and adipose tissue [3], intestine [4] and the liver [5] both in type 1 and type 2 diabetes.

Although the brain does not count as a classical target organ of insulin, it has recently been shown that this polypeptide hormone plays a crucial role in human neurophysiology, and dysregulation of insulin receptor signaling in various mental illnesses [6].

\section{Biomed Central}


It has long been known that insulin can pass the blood brain barrier by receptor mediated endocytosis [7]. Moreover, it turned out that several brain regions are capable of producing insulin in situ [8]. The insulin receptor and insulin receptor substrate-1 (IRS1) are expressed in vegetative nuclei of the hypothalamus, in amygdala, hippocampus and in the neocortex [9]. Based on this expression pattern, cerebral insulin signaling has been implicated in the regulation of neurotransmission, feeding and cognitive functions [10].

Along with leptin, insulin seems to be a negative feedback signal in well-fed state due to its ability to reduce appetite and body weight. It might be assumed that obesity and hyperinsulinism lead to desensitization of insulin receptors situated in the blood brain barrier, giving rise to central insulin resistance [11].

There are several lines of mostly indirect evidence supporting the role of insulin signal transduction in learning and long-term memory. The first observations date back to the famous Rotterdam study, revealing that type 2 diabetes doubled the risk of patients to develop Alzheimertype dementia, while individuals suffering from type 1 diabetes and receiving insulin therapy had four times the risk [12]. These results were corroborated by more recent studies showing that subjects with elevated body mass index, obesity, insulin resistance and diabetes have an increased risk of dementia and cognitive impairment, suggesting a causal link between decreased insulin secretion and the progression of mental decline [13]. Subsequently, post-mortem brain studies unveiled that cerebral insulin, insulin receptor and IGF levels are inversely proportional with the progression of Alzheimer's disease [14]. On the other hand, intranasal and intravenous insulin administration has reportedly improved the cognitive functions of patients suffering from memory disorders, while intracerebroventricular insulin enhanced memory formation in rodents $[15,16]$. Moreover, intracerebral administration of streptozotocin, a drug known to induce type 1 diabetes by impairing pancreatic $\beta$ cells when added intravenously, also led to insulin depletion in the brain with subsequent neurodegeneration [17].

The interrelationship between diabetes and Alzheimer's disease seems to be mutual as neurotoxins termed amyloid beta-derived diffusible ligands have been shown to compromise cerebral insulin signaling [18]. On the other hand, oxidative stress elicited by reactive advanced glycation end products (RAGEs) that are characteristic of diabetes might accelerate neuronal damage in memory disorders [19].

Based on these observations, a group of researchers have recently defined Alzheimer's as a neuroendocrine disorder and coined the terms "type 3 " or "brain-type" diabetes [20], pointing out that this condition can simultaneously be characterized both by central insulin deficiency and insulin resistance. Their work highlighted the importance of impaired insulin signaling in the dysfunction and apoptotic death of cortical neurons.

Although global transcriptome profiling has already been carried out in Alzheimer's disease [21], according to our best knowledge this is the first study aiming to analyze whole genome gene expression profiles of various cerebral areas in streptozotocin-induced and spontaneously diabetic Goto-Kakizaki rats as model animals for type 1 and type 2 diabetes, respectively. Our results demonstrated an altered expression pattern in the hippocampus and prefrontal cortex of type 2 diabetes model, while no such changes were found in the corresponding brain areas of the type 1 model animals.

\section{Results}

The Agilent rat whole genome custom array encompassed 41,129 different oligonucleotide probes according to the latest annotation of the rat genome. Following normalization and technical screening of raw data, approximately $15-26 \%$ of all probes remained. Filtering out genes without significant expression changes resulted in a more drastic reduction of transcript numbers. Statistical analysis and post-screening procedures highlighted spectacular differences in expression profiles of type 2 diabetic brains. Importantly, it turned out that Goto-Kakizaki rats exhibited profound changes in gene expression profiles, while no genes showed significant changes in the transcriptomes of streptozotocin-treated rats versus control animals. Detailed analyses of variations obtained in expression profiles of the studied brain regions of Goto-Kakizaki rats demonstrated large changes in the hippocampus and prefrontal cortex, as 266 versus 147 probes were found to be differentially expressed, respectively, as compared to Wistar controls. Of them, 83 were found in both brain territories. In contrast, only 3 genes with altered expression were identified in the striatum, although they were found in the other two regions as well (Table 1 for detailed gene lists, see Additional File 1). In summary, we obtained a cohort of region-specific or overlapping expression alterations in the Goto-Kakizaki rat model save the striatum that did not show any region-specific patterns at all.

Next, we wished to assign biological relevance to our gene lists by ordering them in biochemical pathways. The Biological Process domain of the Gene Ontology database provided the most extensive pathway assignment. 64 genes from the hippocampus and 36 from the prefrontal cortex were found to be members of certain pathways (Table 1).

Finally, gene expression changes fulfilling the criteria of mathematical-statistical selection and pathway analysis were validated by real time PCR using TaqMan Low Density Arrays. It should be noted that only genes with commercially available TaqMan probes could be analyzed. 
Table 1 Number of genes with significant expression changes in specific brain areas of diabetes models vs. control rats.

\begin{tabular}{lcccccc}
\hline & \multicolumn{3}{c}{$\begin{array}{c}\text { Type 2 diabetes } \\
\text { model }\end{array}$} & \multicolumn{4}{c}{$\begin{array}{c}\text { Type 1 diabetes } \\
\text { model }\end{array}$} \\
& Hipp & Pfc & Str & Hipp & Pfc & Str \\
\hline Statistical analysis & 504 & 232 & 3 & 7 & 0 & 0 \\
Post-screening & 266 & 147 & 3 & 0 & 0 & 0 \\
Genes in significant pathways & 64 & 36 & 0 & 0 & 0 & 0 \\
Genes to be validated* & 42 & 27 & 0 & 0 & 0 & 0 \\
Validated genes & $\mathbf{3 0}$ & $\mathbf{2 2}$ & 0 & 0 & 0 & 0 \\
\hline
\end{tabular}

The table represents significant genes remaining following each stage of the normalization-evaluation procedure. For details, see Methods and Results sections. Abbreviations used: Hipp: hippocampus; Pfc: prefrontal cortex; Str: striatum.

* Reduction was due to technical criteria of the TaqMan RT-PCR system (only genes with commercially available TaqMan probes could be validated).

Therefore, 42 out of the 64 hippocampal and 27 out of the 36 prefrontal genes were subject to validation. Finally, 30 genes from the hippocampus (71\%) and 22 genes from the prefrontal cortex $(82 \%)$ were validated (Table 2; for detailed gene lists, see Additional File 2). According to our results, 9 genes showed changes both in the hippocampus and in prefrontal cortex in the type 2 diabetes model (for a detailed list, see Additional File 2). Finally, pathway analysis revealed that most genes with altered expression patterns in the hippocampus are involved in oxidative stress and DNA damage signaling, cell cycle regulation, development and lipid metabolism of the central nervous system as well as in the regulation of feeding behavior (Table 2 and Figure 1).

Regarding the prefrontal cortex, perturbed expression of a set of neurotransmission and lipid metabolism related genes has been unveiled with significant overlap with the hippocampal alterations (Table 3 Additional File 2 and Figure 1). These findings seem to be consistent with functional cerebral impairments described in diabetic individuals such as cognitive deficit, increased appetite and food ingestion, and development of depression [22]. It would be of importance to clarify whether genes with altered expression patterns are controlled by insulindependent transcription factors such as members of the forkhead (FOXO) family [23].

\section{Discussion}

Insulin regulates gene expression via a set of transcription factors including the FOXO family [24]. As insulin and its receptors are both known to be expressed and to govern important functions in the brain, it seemed reasonable to search for altered gene expression patterns in animal models of type 1 and type 2 diabetes characterized by absolute or relative insulin deficiency. Here we demonstrated a substantial difference in the gene expression pattern of type 2 diabetic rats vs. control animals.
The genetically determined, spontaneously diabetic Goto-Kakizaki rats exhibited profound gene expression alterations suggesting that long-standing impairment of insulin signaling has a well detectable effect on the central nervous system. On the other hand, we could hardly detect any alterations in the streptozotocin-induced diabetic animal model (Table 1), suggesting that acute insulin deficiency and/or elevated blood sugar levels do not influence significantly the cerebral gene expression pattern, or at least it is undetectable four weeks after the streptozotocin treatment in a microarray based experiment. It is tempting to speculate that streptozotocininduced diabetic rats might successfully compensate peripheral insulin deficiency by increased cerebral insulin production. However, this presumption seems to contradict the fact that activation of the ins 2 gene was not detected - maybe due to low sensitivity of the whole genome custom array.

Three main brain regions have been studied here: the prefrontal cortex and hippocampus were analyzed due to their well-known roles in learning and memory formation, while the striatum seemed to be an easily dissectable control region where no insulin action had been presumed. It is also interesting to note that streptozotocintreted rats exhibited some gene expression alterations in the hippocampus only. These observations are in a good agreement with the findings of Agrawal et al., showing that insulin and its receptor are mostly expressed in this brain region, and intracerebroventricular administration of streptozotocin induced memory deficit in rats [25].

Streptozotocin has been proven to induce insulin deficiency and hyperglycemia ( $\geq 15 \mathrm{mM}$ ) within 72 hours in treated animals, and they were alive for 4 weeks following beta-cell destruction. In our opinion, this time window should have been enough to alter gene expression profiles in the brain as there are several reports highlighting the early effects of streptozotocin on gene expression in various organs [26]. The major drawback of the global microarray method is its minor sensitivity compared to that of TaqMan-based quantitative reverse transcription PCR assays. However, the high RT-PCR validation rate of microarray data in Goto-Kakizaki rats (71\% in the hippocampus and $82 \%$ in the prefrontal cortex, respectively) convinced us of the reasonably good reliability of the chip hybridization technique. Theoretically, some minor gene expression alterations in the brains of type 1 diabetic model animals might have been left undetected by the chip hybridization technique, therefore, we are committed to validate the "non-changed" status of a set of genes which were significantly altered in type 2 diabetic animals using open-array real-time PCR assays.

Analyzing the specific genes, the mRNA levels of galanin, an inhibitory neuropeptide with pleiotropic roles were substantially upregulated in the hippocampus. 
Table 2 List of significant pathways in the hippocampus of type 2 diabetic rats.

\begin{tabular}{|c|c|c|c|}
\hline GO Biological processes & HIPPOCAMPUS & Validated & Not validated \\
\hline \multirow[t]{2}{*}{ Insulin/GH secretion } & GO:30073: insulin secretion & Gal & \\
\hline & GO:30252: growth hormone secretion & Gal & \\
\hline \multirow{10}{*}{$\begin{array}{l}\text { Oxidative stress DNA } \\
\text { damage cell cycle }\end{array}$} & GO:6950: response to stress & Gal & \\
\hline & GO:305: response to oxygen radical & $\mathrm{CxCl} 4(\mathrm{Pf} 4)$ & \\
\hline & GO:303: response to superoxide & Akap3 & \\
\hline & GO:302: response to reactive oxygen species & Gal & Nudt15_predicted \\
\hline & GO:15992: proton transport & Ucp2 & \\
\hline & $\begin{array}{l}\text { GO:6977: DNA damage response, signal transduction by p53 class } \\
\text { mediator resulting in cell cycle arrest }\end{array}$ & Ptprv & \\
\hline & GO:42770: DNA damage response, signal transduction & Ftcd & \\
\hline & GO:7346: regulation of progression through mitotic cell cycle & Snf1lk & \\
\hline & GO:6269: DNA replication, synthesis of RNA primer & $\begin{array}{l}\text { NM_001008768 } \\
\text { (Prim1) }\end{array}$ & \\
\hline & GO:7089: traversing start control point of mitotic cell cycle & Cdk10 & \\
\hline \multirow[t]{2}{*}{ Lipid metabolism } & GO:1573: ganglioside metabolism & Gm2a & \\
\hline & GO:6695: cholesterol biosynthesis & Acaa2 & Acaa2 \\
\hline \multirow[t]{2}{*}{ Eating/feeding behavior } & GO:7631: feeding behavior & Gal, Agrp & \\
\hline & GO:42755: eating behavior & Agrp & Stat3 \\
\hline \multirow[t]{2}{*}{$\begin{array}{l}\text { Development of the } \\
\text { nervous system }\end{array}$} & GO:7399: nervous system development & $\begin{array}{l}\text { Gal, Mobp, } \\
\text { Mobp, Cntn3 }\end{array}$ & $\begin{array}{l}\text { Ednrb, RGD1311340_predicted, } \\
\text { Stat3, XM_242005 }\end{array}$ \\
\hline & GO:7422: peripheral nervous system development & Sncg & Ednrb \\
\hline \multirow[t]{9}{*}{ Others } & GO:50776: regulation of immune response & Gal, II22ra2 & \\
\hline & GO:6952: defense response & $M \times 2$ & \\
\hline & GO:7194: negative regulation of adenylate cyclase activity & Grm2 & \\
\hline & GO:6032: chitin catabolism & Chi3l1 & \\
\hline & GO:42572: retinol metabolism & Retsat & \\
\hline & GO:45123: cellular extravasation & Itgam & \\
\hline & GO:19637: organophosphate metabolism & Pter & \\
\hline & GO:6928: cell motility & Akap3, Grm2 & Stat3 \\
\hline & GO:9615: response to virus & M×2, Oas1 & XM_215121 \\
\hline
\end{tabular}

Data were obtained using the GO pathway analysis software; specific GO pathway identification numbers are provided in the second column. "Validated genes" were confirmed by quantitative PCR analysis. Validated genes found in more than 2 significant pathways are shown in bold.

Notably, galanin were identified in almost all perturbed pathways of the hippocampus (Table 2). Our results corroborated the findings of Mei et al. who detected elevated galanin expression in the celiac ganglion in diabetic rats [27]. Intracerebroventricular administration of galanin or its overexpression in transgenic mice was shown to compromise hippocampus-dependent learning processes $[28,29]$. Galanin has been proposed to play a role in depression-like behavior [30]. On the other hand, improvement of cognitive functions has been reported in animals treated with galanin receptor antagonists [28]. As cerebral insulin deficiency presents with similar symptoms, it is tempting to speculate that impairment of cerebral functions in diabetes might be mediated at least in part by elevated galanin levels. This assumption is supported by the fact that plasma galanin levels have been found to be significantly elevated in patients with type 2 diabetes [31], and increased plasma galanin levels were measured following oral glucose load in a healthy population [32]. If we managed to find a causal relationship between cerebral insulin deficiency and galanin overexpression, we might be able to ameliorate cerebral symptoms of diabetes via pharmacological modulation of galanin receptors and to slow down the progression of type 3 diabetes [20].

The role of galanin receptors is also highlighted by our results which demonstrated altered galanin receptor 2 expression levels in the prefrontal cortex (Table 3 ). Type 2 galanin receptors are mostly expressed in the perikaryon of neurons, mediating calcium signals and promoting the survival of neurons [33], and their stimulation reportedly elicited antidepressive effects [34].

Apart from galanin and its receptor, there are several other validated genes as well, which have already been implicated in the pathogenesis of both diabetes and psychiatric disorders in some respect. For instance, Chi3l1 


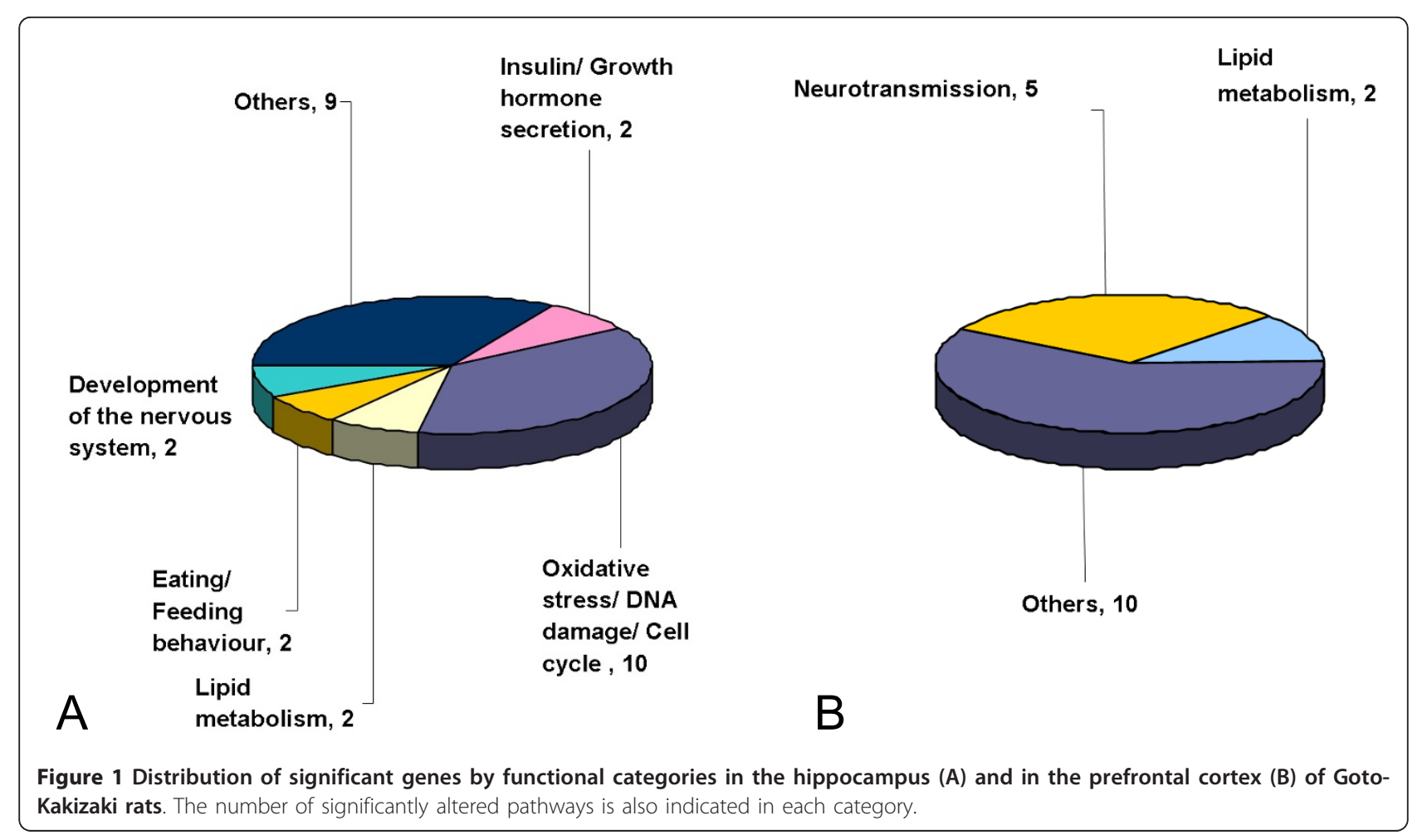

( $Y K L-40$, chitinase 3 -like 1 ) has recently been shown to represent an obesity-independent novel marker of type 2 diabetes [35]. On the other hand, Chi3l1 has been regarded as a schizophrenia susceptibility gene, a mediator of stress-induced cellular responses [36]. SNCG (synuclein gamma) has recently been termed an adipocyte-neuron gene that is coordinately expressed with leptin in human obesity and might promote adipocyte differentiation [37]. Apart from its well-known role in the development of neurodegenerative diseases [38], SNCG has also been

Table 3 List of significant pathways in the prefrontal cortex of type 2 diabetic rats.

\begin{tabular}{|c|c|c|c|}
\hline GO Biological processes & PREFRONTAL CORTEX & Validated & Not validated \\
\hline \multirow[t]{5}{*}{ neurotransmission } & GO:7611: learning and/or memory & Galr2, Prkcc, Gm2a & \\
\hline & GO:7268: synaptic transmission & Galr2, Prkcc, Grm2 & \\
\hline & GO:1507: acetylcholine catabolism in synaptic cleft & Colq & \\
\hline & GO:1504: neurotransmitter uptake & Slc17a6 & \\
\hline & GO:17158: regulation of calcium ion-dependent exocytosis & Trpv6 & \\
\hline \multirow[t]{2}{*}{ lipid metabolism } & GO:1573: ganglioside metabolism & Gm2a & \\
\hline & GO:45332: phospholipid translocation & Abca1 & \\
\hline \multirow[t]{10}{*}{ others } & GO:9649: entrainment of circadian clock & Bhlhb2 & \\
\hline & GO:8228: opsonization & $\mathrm{Cd} 47$ & \\
\hline & GO:6032: chitin catabolism & Chi3l1 & \\
\hline & GO:6547: histidine metabolism & Ftcd & \\
\hline & GO:7497: posterior midgut development & Ret & \\
\hline & GO:30277: maintenance of gastrointestinal epithelium & Tff1 & \\
\hline & GO:6936: muscle contraction & Galr2, Lsp1 & Sgca_predicted \\
\hline & GO:19882: antigen presentation & NM_001008842, RT1-Aw2 (Y13890) & \\
\hline & GO:9615: response to virus & Oas1 & XM_215121 \\
\hline & GO:7635: chemosensory behavior & Prkcc, Prkce & Prkce \\
\hline
\end{tabular}

Data were obtained using the GO pathway analysis software; specific GO pathway identification numbers are provided in the second column. "Validated genes" were confirmed by quantitative PCR analysis. Validated genes found in more than 2 significant pathways are shown in bold. 
implicated in depression [39], dopamine release [40] and as an interacting partner of the dopamine transporter in rats [41].

Perturbation of brain signaling pathways could also be a very important hallmark of type 2 diabetes. Here we identified three genes of cerebral signaling (protein kinase $\mathrm{C}$ gamma and epsilon, and the RET tyrosine kinase) with altered cerebral expression profiles in GotoKakizaki rats. They have been shown to play a pathophysiological role in brain dysfunction previously. For instance, expression of the neuron-specific gamma isoform of protein kinase $\mathrm{C}$ (Prkcc) that has been implied in the regulation of learning and memory formation (Additional File 2) was more than twofold upregulated in the prefrontal cortex of Goto-Kakizaki rats (Additional File 2). Schlaepfer et al. demonstrated that certain polymorphisms of the Prkcc gene are associated with behavioral disinhibition and attention deficit hyperactivity disorder (ADHD) in humans, while PKC-gamma deficient mice exhibited impulsivity, anxiety and increased ethanol consumption [42]. Importantly, the epsilon isoform of PKC (Prkce) is also overexpressed in the type 2 diabetic model (Additional File 2). This kinase is reportedly involved in neuronal ion channel activation, apoptosis and insulin exocytosis. Recently, Prkce has been implicated in the loss of insulin secretory responsiveness during the development of type 2 diabetes [43], while others highlighted its role in the pathomechanism of drug dependence and addiction [44]. Shelton et al. revealed decreased Prkce protein levels in post mortem brain specimens of patients with major depression [45]. Finally, we demonstrated changes in the expression level of the $R E T$ protooncogene, a receptor tyrosine kinase containing cadherin-like repeats in its extracellular domain, that plays a pivotal role in neural crest development. Mutations in this gene might elicit multiple endocrine neoplasia type $2 \mathrm{~B}$ with diabetes [46]. Interestingly, $R E T$ activity has been shown to modulate and shape the brain dopaminergic systems which are known mediators of several personality traits [47].

As far as the theory of type 3 diabetes is concerned, our microarray data revealed a couple of genes which might provide a link between diabetes and neurodegeneration. Apart from the already mentioned synuclein gamma, uncupling protein 2 (UCP2), the $\mathrm{ABC}$-transporter $A B C A 1$ and the cell surface antigen $C D 47$ should also be mentioned in this context. UCP2, a well-known inner mitochondrial membrane protein, responsible for energy dissipation and heat production, has been found to associate with obesity, diabetes and regulation of insulin secretion [48]. On the other hand, the UCP2 gene is induced in a ghrelin-dependent fashion and protects from neurodegeneration [49]. UCP2 expression was significantly downregulated in the hippocampus of our type 2 diabetic rat model (Additional File 1), implying that its neuroprotective effect might be absent from the diabetic brain.

Mutations in the cholesterol efflux pump $A B C A 1$ have been associated with Tangier's disease. Beyond that, $A B C A 1$ has been implicated in insulin secretion from pancreatic beta cells [50], and some single nucleoide polymorphisms (SNPs) of this gene have been demonstrated to associate with dementia (rs2230805) [51] and Alzheimer's disease (rs1800977 and rs2422493) [52]. We found significant downregulation of $A B C A 1$ levels in the prefrontal cortex of Goto-Kakizaki rats (Additional File 2); hence it seems logical to assume that elevated cytosolic cholesterol levels might impair the viability of neurons via affecting membrane fluidity.

The gene for $C D 47$ encodes a membrane protein which is involved in the increase in intracellular calcium concentration that occurs upon cell adhesion to the extracellular matrix. There is ample evidence supporting the role of $C D 47$ in pancreatic insulin secretion [53]. Moreover, $C D 47$ has been shown to interact with amyloid beta peptide in Alzheimer's disease [54]. We measured elevated CD47 mRNA levels both in the hippocampus and in the prefrontal cortex of type 2 diabetes model animals, providing a plausible link between central insulin resistance and Alzheimer-type neurodegeneration.

\section{Conclusion}

In conclusion, our study shed light on the seminal role of insulin in maintaining the functions of the central nervous system by unveiling characteristic perturbations in cerebral gene expression profiles in type 2 diabetic rats. We identified several cerebral expression changes in genes which were previously assumed to play a role in pancreatic insulin secretion, implying that these genes might mediate insulin production and exocytosis in the brain as well. Our results should prompt further investigations to decipher insulin signaling pathways in the brain and a detailed analysis of the transcriptional regulation of diabetes-associated genes having been identified in this study.

\section{Methods}

\section{Animals}

Experiments were performed on ten-week old male rats (weighing $286 \pm 60 \mathrm{~g}$ ). Streptozotocin-treated inbred white Wistar rats were used as model animals for type 1 diabetes, and Goto-Kakizaki rats were the polygenic nonobese models of type 2 diabetes [55]. Wistar rats at 6 weeks of age, weighing approximately $170 \mathrm{~g}$, were injected with $65 \mathrm{mg} /$ body mass $\mathrm{kg}$ streptozotocin intravenously. The development of diabetes was confirmed by elevated fasting blood sugar levels $(\geq 15 \mathrm{mM}$ measured $72 \mathrm{hrs}$ following the injection), and the streptozotocin-treated rats 
were sacrificed by cervical dislocation 4 weeks after the injection. Diabetic animals as well as their age- and body mass matched Wistar controls and age-matched GotoKakizaki rats were kept on normal chow. All experimental protocols were in accordance with the guidelines of the Committee on the Care and Use of Laboratory Animals of the Council on Animal Care at the Semmelweis University, Budapest, Hungary (ethical permission No.: TUKEB 99/94).

\section{Tissue harvesting}

9 animals from each group at 10 weeks of age were anaesthetized with phenobarbital and killed by decapitation. The brain was removed and the striatum, hippocampus and prefrontal cortex were dissected. Samples from 3-3 identically treated animals were pooled. That means, 3 biological parallels were prepared from each brain region of type 1 or type 2 diabetic and control animals, amounting to a total of 27 different pooled samples. Excised tissue samples were immediately fixed in RNAlater RNA stabilization reagent (Qiagen).

\section{Sample preparation and oligonucleotide microarray hybridization}

Total RNA was extracted from samples by homogenization using the RNeasy Kit (Qiagen), according to the manufacturer's instructions. RNA integrity and purity were checked both by agarose gel electrophoresis and with an Agilent 2100 Bioanalyzer. Samples of acceptable quality fulfilled the following criteria: $\mathrm{OD}_{260 / 280}>1.8, \mathrm{OD}_{260 / 230}>1.8$ and RIN $>7$. Reverse transcription was performed using 1000 ng of total RNA from each sample. Labeling of single-stranded cRNA, hybridization and scanning were carried out at the Microarray Core Facility of the Department of Genetics, Cell- and Immunobiology of Semmelweis University, using Agilent's One-Color Microarray-Based Gene Expression Analysis Protocol, Version 5.5 (G414090040). Labeling of samples was performed with Agilent's Low RNA Input Linear Amplification Kit PLUS assay using the Cy3 dye. Dye incorporation was controlled by a Nanodrop spectrophotometer; all samples were labeled with an efficiency of 10.2 - $17.5 \mathrm{pmol} \mathrm{Cy} 3 / \mu \mathrm{g}$ cRNA. $1650 \mathrm{ng}$ of cRNA were hybridized to Agilent's Rat Whole Genome Custom Arrays. Arrays were run on all 27 biological samples. Hybridized arrays were imaged with Agilent's Microarray Scanner, Agilent Feature Extraction Software version 9.1 in the extended dynamic range at $100 \%$ and $10 \%$ laser beam intensities at a resolution of $5 \mu \mathrm{m}$.

\section{Data analysis}

Data analysis was performed using the GeneSpring GX software (Agilent Technologies, version 7.3). For normalization, the samples were grouped according to brain areas. In this way, gene expression data from treated samples in groups were normalized to the median of control samples of each group. As quality control, genes with poor hybridization signals (flag screening) and those with unaltered expression (not showing a minimum of 2 -fold difference between their maximal and minimal expression levels under any conditions) were excluded from subsequent analysis. Statistical analysis of data obtained from the normalization and screening procedures was performed to select probes with at least a twofold, statistically significant expression alteration in type 1 or type 2 diabetic animals compared to Wistar controls using Welch's $t$-test supplemented with the Benjamini-Hochberg multiple correction test with a $\mathrm{p}=0.05$ cutoff. Finally, a post-screening procedure was implemented to exclude false positive probes, i.e. signals with "absent" flag in at least 2 out of 3 biological replicates, and those with raw intensity signals less than 100 arbitrary units.

The Gene Ontology database (URL: http://www.geneontology.org)was used to assign biological relevance to our data and to identify genes by ordering them in relevant biochemical pathways. Biochemical pathways were regarded significantly altered if they comprised a significant number of genes from our lists $(\mathrm{p}<0.05)$.

\section{Validation by real-time PCR}

Genes that fulfilled the criteria of technical, statistical and pathway analyses were validated by the quantitative reverse transcription PCR-based TaqMan Low Density Array (Applied Biosystems) system, according to the manufacturer's protocol. cDNA samples for this test were synthesized from the same RNA samples that had been prepared for microarray hybridization. Relative gene expression data were obtained using the 2(-Delta Delta $\mathrm{C}_{\mathrm{T}}$ ) method described by Livak and Schmittgen in detail [56].

Briefly, six genes were selected as potential housekeeping (internal control) genes for normalization of RT-PCR data [histone deacetylase 3 (Hdac3), ATP-citrate lyase (Acly), beta-actin (Actb), beta-2 microglobulin (B2m), TATA box binding protein $(T b p), 18 \mathrm{~S}$ ribosomal RNA $(18 S)$ ]. By cross-checking their relative expression levels and scattering scores, we chose the following 3 genes with most stable and constant expression: $\mathrm{Hdac} 3, \mathrm{Tbp}$ and $B 2 \mathrm{~m}$. The expression of all target genes was normalized to the mean of the expression of the housekeeping genes (relative quantification). Cycle threshold $\left(\mathrm{C}_{\mathrm{T}}\right)$ values were set in the exponential range of the amplification plots using the 7300 System Sequence Detection Software 1.3. $\Delta \Delta \mathrm{C}_{\mathrm{T}}$-values corresponded to the difference between the $C_{\mathrm{T}}$-values of the genes examined and those of the arithmetical mean of the expression of the 3 housekeeping calibrator (internal control) genes. Relative expression levels of genes were calculated and expressed 
as $2^{-\Delta \Delta C T}$. Finally, the Mann-Whitney test $(\mathrm{p}<0.01)$ was used for statistical analysis of qRT-PCR data.

\section{Data deposition}

The data discussed in this publication have been deposited in NCBI's Gene Expression Omnibus (Edgar et al., 2002) and are accessible through GEO Series accession number GSE34451 (http://www.ncbi.nlm.nih.gov/geo/ query/acc.cgi?acc $=$ GSE34451).

\section{Additional material}

Additional file 1: List of differentially expressed genes in the brain areas of Goto-Kakizaki rats. Genes are ordered according to their fold expression changes. Genes are identified both by gene name, Genbank accession number and gene symbol. The file contains four table sheets displaying genes with significantly altered expression levels (more than twofold or less than 0.5 fold) in the hippocampus only ("Hippocampus", 180 genes), in the prefrontal cortex only ("Prefrontal cortex", 61 genes), both in hippocampus and prefrontal cortex ("Hipp\&Pfc", 83 genes) and both in hippocampus, prefrontal cortex and striatum ("Hipp\&Pfc\&Str", 3 genes), respectively. In the corporate lists genes are ordered according to their fold expression changes observed in the hippocampus.

Additional file 2: List of validated genes in the brain areas of GotoKakizaki rats. Genes are shown in alphabetical order of gene symbols. Genes are identified both by gene name, Genbank accession number and gene symbol. The file contains three table sheets displaying genes with RT-PCR validated, significantly altered expression levels (more than twofold or less than 0.5 fold) in the hippocampus only ("Hippocampus", 30 genes), in the prefrontal cortex only ("Prefrontal cortex", 22 genes), both in hippocampus and prefrontal cortex ("Hipp\&Pfc", 9 genes), respectively. In the corporate lists genes are ordered according to their fold expression changes observed in the hippocampus.

\section{Acknowledgements}

The work presented here has been supported by the Hungarian funds OTKA K 83766 and ETT 258_09.

\section{Author details}

${ }^{1}$ Department of Medical Chemistry, Molecular Biology and

Pathobiochemistry, Semmelweis University, Budapest, Hungary. ${ }^{2}$ Department of Pharmacology, Institute of Experimental Medicine, Hungarian Academy of Sciences, Budapest, Hungary.

\section{Authors' contributions}

OA performed the data and statistical analysis; MS conceived of the study, participated in its design and coordinated the study; AV and KR participated in the design of the study and selection of animals; BKS performed brain dissections and tissue harvesting, EK participated in mRNA preparation; GK participated in study design and drafted the manuscript. All authors read and approved the final manuscript.

Received: 1 June 2011 Accepted: 27 February 2012

Published: 27 February 2012

\section{References}

1. Musi N, Goodyear LJ: Insulin resistance and improvements in signal transduction. Endocrine 2006, 29:73-80.

2. Mounier C, Posner Bl: Transcriptional regulation by insulin: from the receptor to the gene. Can J Physiol Pharmacol 2006, 84:713-24.

3. Yang YL, Xiang RL, Yang C, Liu XJ, Shen WJ, Zuo J, Chang YS, Fang FD: Gene expression profile of human skeletal muscle and adipose tissue of Chinese Han patients with type 2 diabetes mellitus. Biomed Environ Sci 2009, 22:359-68.
4. Sun J, Wang D, Jin T: Insulin alters the expression of components of the Wnt signaling pathway including TCF-4 in the intestinal cells. Biochim Biophys Acta 2010, 1800:344-51.

5. Matsumoto K, Yokoyama SI: Gene expression analysis on the liver of cholestyramine-treated type 2 diabetic model mice. Biomed Pharmacother 2010.

6. Huang CC, Lee CC, Hsu KS: The role of insulin receptor signaling in synaptic plasticity and cognitive function. Chang Gung Med J 2010, 33:115-25.

7. Baura GD, Foster DM, Kaiyala K, Porte D Jr, Kahn SE, Schwartz MW: Insulin transport from plasma into the central nervous system is inhibited by dexamethasone in dogs. Diabetes 1996, 45:86-90.

8. Devaskar SU, Giddings SJ, Rajakumar PA, Carnaghi LR, Menon RK, Zahm DS: Insulin gene expression and insulin synthesis in mammalian neuronal cells. J Biol Chem 1994, 269:8445-54.

9. Baskin DG, Schwartz MW, Sipols AJ, D'Alessio DA, Goldstein BJ, White MF: Insulin receptor substrate-1 (IRS-1) expression in rat brain. Endocrinology 1994, 134:1952-5.

10. Wada A, Yokoo H, Yanagita T, Kobayashi H: New twist on neuronal insulin receptor signaling in health, disease, and therapeutics. J Pharmacol Sci 2005, 99:128-43.

11. Kern W, Benedict C, Schultes B, Plohr F, Moser A, Born J, Fehm HL, Hallschmid M: Low cerebrospinal fluid insulin levels in obese humans. Diabetologia 2006, 49:2790-2.

12. Ott A, Stolk RP, van Harskamp F, Pols HA, Hofman A, Breteler MM: Diabetes mellitus and the risk of dementia: The Rotterdam Study. Neurology 1999, 53:1937-42.

13. Rönnemaa E, Zethelius B, Sundelöf J, Sundström J, DegermanGunnarsson M, Berne C, Lannfelt L, Kilander L: Impaired insulin secretion increases the risk of Alzheimer disease. Neurology 2008, 71:1065-71.

14. Kroner Z: The relationship between Alzheimer's disease and diabetes: Type 3 diabetes? Altern Med Rev 2009, 14:373-9.

15. Benedict C, Kern W, Schultes B, Born J, Hallschmid M: Differential sensitivity of men and women to anorexigenic and memory-improving effects of intranasal insulin. J Clin Endocrinol Metab 2008, 93:1339-44.

16. Park CR, Seeley RJ, Craft S, Woods SC: Intracerebroventricular insulin enhances memory in a passive-avoidance task. Physiol Behav 2000, 68:509-14.

17. Lester-Coll N, Rivera EJ, Soscia SJ, Doiron K, Wands JR, de la Monte SM: Intracerebral streptozotocin model of type 3 diabetes: relevance to sporadic Alzheimer's disease. J Alzheimers Dis 2006, 9:13-33.

18. Viola KL, Velasco PT, Klein WL: Why Alzheimer's is a disease of memory: the attack on synapses by A beta oligomers (ADDLs). I Nutr Health Aging 2008, 12:51S-7S.

19. Valente $T$, Gella A, Fernàndez-Busquets $X$, Unzeta M, Durany N: Immunohistochemical analysis of human brain suggests pathological synergism of Alzheimer's disease and diabetes mellitus. Neurobiol Dis 2010, 37:67-76.

20. de la Monte SM, Wands JR: Alzheimer's disease is type 3 diabetesevidence reviewed. J Diabetes Sci Technol 2008, 2:1101-13.

21. Tan MG, Chua WT, Esiri MM, Smith AD, Vinters HV, Lai MK: Genome wide profiling of altered gene expression in the neocortex of Alzheimer's disease. J Neurosci Res 2010, 88:1157-69.

22. Janocha A, Bolanowski M, Pilecki W, Małyszczak K, Salomon E, Woźniak W, Skalik R, Tumińska A, Kałka D, Sobieszczańska M: Cognitive disorders in type 2 diabetic patients with recognized depression. Neuro Endocrinol Lett 2010, 31:399-405.

23. Gross DN, Wan M, Birnbaum MJ: The role of FOXO in the regulation of metabolism. Curr Diab Rep 2009, 9:208-14.

24. Cheng Z, White MF: Targeting Forkhead box 01 from the concept to metabolic diseases: lessons from mouse models. Antioxid Redox Signal 2011, 14:649-61.

25. Agrawal $R$, Tyagi $E$, Shukla $R$, Nath $C$ : Insulin receptor signaling in rat hippocampus: A study in STZ (ICV) induced memory deficit model. Eur Neuropsychopharmacol 2011, 21:261-73.

26. Hulmi JJ, Silvennoinen M, Lehti M, Kivelä R, Kainulainen H: Altered REDD1, myostatin and Akt/mTOR/FoxO/MAPK Signaling in Streptozotocininduced Diabetic Muscle Atrophy. Am J Physiol Endocrinol Metab 2011.

27. Mei Q, Mundinger TO, Lernmark K, Taborsky GJ Jr: Increased galanin expression in the celiac ganglion of BB diabetic rats. Neuropeptides 2006, 40:1-10. 
28. Ogren SO, Kehr J, Schött PA: Effects of ventral hippocampal galanin on spatial learning and on in vivo acetylcholine release in the rat. Neuroscience 1996, 75:1127-40.

29. Steiner RA, Hohmann JG, Holmes A, Wrenn CC, Cadd G, Juréus A, Clifton DK, Luo M, Gutshall M, Ma SY, Mufson EJ, Crawley JN: Galanin transgenic mice display cognitive and neurochemical deficits characteristic of Alzheimer's disease. Proc Natl Acad Sci USA 2001, 98:4184-9.

30. Kuteeva E, Wardi T, Hökfelt T, Ogren SO: Galanin enhances and a galanin antagonist attenuates depression-like behaviour in the rat. Eur Neuropsychopharmacol 2007, 17:64-9.

31. Legakis I, Mantzouridis T, Mountokalakis T: Positive correlation of galanin with glucose in type 2 diabetes. Diabetes Care 2005, 28:759-60.

32. Legakis IN, Mantzouridis T, Mountokalakis T: Positive correlation of galanin with glucose in healthy volunteers during an oral glucose tolerance test. Horm Metab Res 2007, 39:53-5.

33. Elliott-Hunt CR, Pope RJ, Vanderplank P, Wynick D: Activation of the galanin receptor 2 (GalR2) protects the hippocampus from neuronal damage. J Neurochem 2007, 100:780-9.

34. Kuteeva $E$, Wardi $T$, Lundström $L$, Sollenberg $U$, Langel $U$, Hökfelt $T$, Ogren SO: Differential role of galanin receptors in the regulation of depression-like behavior and monoamine/stress-related genes at the cell body level. Neuropsychopharmacology 2008, 33:2573-85.

35. Nielsen AR, Erikstrup $C$, Johansen JS, Fischer CP, Plomgaard P, KroghMadsen R, Taudorf S, Lindegaard B, Pedersen BK: Plasma YKL-40: a BMIindependent marker of type 2 diabetes. Diabetes 2008, 57:3078-82.

36. Yang MS, Morris DW, Donohoe G, Kenny E, O'Dushalaine $C T$, Schwaiger $\mathrm{S}$, Nangle JM, Clarke S, Scully P, Quinn J, Meagher D, Baldwin P, Crumlish N, O'Callaghan E, Waddington JL, Gill M, Corvin A: Chitinase-3-like 1 (CHI3L1) gene and schizophrenia: genetic association and a potential functional mechanism. Biol Psychiatry 2008, 64:98-103.

37. Oort PJ, Knotts TA, Grino M, Naour N, Bastard JP, Clément K, Ninkina N, Buchman VL, Permana PA, Luo X, Pan G, Dunn TN, Adams SH: Gammasynuclein is an adipocyte-neuron gene coordinately expressed with leptin and increased in human obesity. J Nutr 2008, 138:841-8.

38. Ninkina N, Peters O, Millership S, Salem H, van der Putten H, Buchman VL: Gamma-synucleinopathy: neurodegeneration associated with overexpression of the mouse protein. Hum Mol Genet 2009, 18:1779-94.

39. Wersinger C, Sidhu A: Partial regulation of serotonin transporter function by gamma-synuclein. Neurosci Lett 2009, 453:157-61.

40. Senior SL, Ninkina N, Deacon R, Bannerman D, Buchman VL, Cragg SJ, Wade-Martins R: Increased striatal dopamine release and hyperdopaminergic-like behaviour in mice lacking both alpha-synuclein and gamma-synuclein. Eur J Neurosci 2008, 27:947-57.

41. Boyer F, Dreyer JL: The role of gamma-synuclein in cocaine-induced behaviour in rats. Eur J Neurosci 2008, 27:2938-51.

42. Schlaepfer IR, Clegg HV, Corley RP, Crowley TJ, Hewitt JK, Hopfer CJ, Krauter K, Lessem J, Rhee SH, Stallings MC, Wehner JM, Young SE, Ehringer MA: The human protein kinase $\mathrm{C}$ gamma gene (PRKCG) as a susceptibility locus for behavioral disinhibition. Addict Biol 2007, 12:200-9.

43. Biden TJ, Schmitz-Peiffer C, Burchfield JG, Gurisik E, Cantley J, Mitchell CJ, Carpenter $\mathrm{L}$ : The diverse roles of protein kinase $\mathrm{C}$ in pancreatic beta-cell function. Biochem Soc Trans 2008, 36:916-9.

44. Olive $M F$, Messing RO: Protein kinase $\mathrm{C}$ isozymes and addiction. $\mathrm{Mol}$ Neurobiol 2004, 29:139-54.

45. Shelton RC, Hal Manier D, Lewis DA: Protein kinases $A$ and $C$ in postmortem prefrontal cortex from persons with major depression and normal controls. Int I Neuropsychopharmacol 2009, 12:1223-32.

46. Donckier JE, Rosière A, Heureux E, Michel L: Diabetes mellitus as a primary manifestation of multiple endocrine neoplasia type 2B. Acta Chir Belg 2008, 108:732-7.

47. Mijatovic J, Airavaara M, Planken A, Auvinen P, Raasmaja A, Piepponen TP, Costantini F, Ahtee L, Saarma M: Constitutive Ret activity in knock-in multiple endocrine neoplasia type B mice induces profound elevation of brain dopamine concentration via enhanced synthesis and increases the number of TH-positive cells in the substantia nigra. J Neurosci 2007, 27:4799-809.

48. González-Barroso MM, Giurgea I, Bouillaud F, Anedda A, BellannéChantelot C, Hubert L, de Keyzer Y, de Lonlay P, Ricquier D: Mutations in UCP2 in congenital hyperinsulinism reveal a role for regulation of insulin secretion. PLoS One 2008, 3:e3850.
49. Andrews ZB, Erion D, Beiler R, Liu ZW, Abizaid A, Zigman J, Elsworth JD, Savitt JM, DiMarchi R, Tschoep M, Roth RH, Gao XB, Horvath TL: Ghrelin promotes and protects nigrostriatal dopamine function via a UCP2dependent mitochondrial mechanism. J Neurosci 2009, 29:14057-65.

50. Koseki M, Matsuyama A, Nakatani K, Inagaki M, Nakaoka H, Kawase R, YuasaKawase M, Tsubakio-Yamamoto K, Masuda D, Sandoval JC, Ohama T, Nakagawa-Toyama Y, Matsuura F, Nishida M, Ishigami M, Hirano K, Sakane N, Kumon Y, Suehiro T, Nakamura T, Shimomura I, Yamashita S: Impaired insulin secretion in four Tangier disease patients with ABCA1 mutations. J Atheroscler Thromb 2009, 16:292-6.

51. Reynolds CA, Hong MG, Eriksson UK, Blennow K, Bennet AM, Johansson B, Malmberg B, Berg S, Wiklund F, Gatz M, Pedersen NL, Prince JA: A survey of $A B C A 1$ sequence variation confirms association with dementia. Hum Mutat 2009, 30:1348-54.

52. Rodríguez-Rodríguez E, Vázquez-Higuera JL, Sánchez-Juan P, Mateo I, Pozueta A, Martínez-García A, Frank A, Valdivieso F, Berciano J, Bullido MJ, Combarros $\mathrm{O}$ : Epistasis between intracellular cholesterol traffickingrelated genes (NPC1 and ABCA1) and Alzheimer's disease risk. J Alzheimers Dis 2010, 21:619-25.

53. Kobayashi M, Ohnishi $H$, Okazawa H, Murata $Y$, Hayashi $Y$, Kobayashi $H_{\text {, }}$ Kitamura T, Matozaki T: Expression of Src homology 2 domain-containing protein tyrosine phosphatase substrate- 1 in pancreatic beta-Cells and its role in promotion of insulin secretion and protection against diabetes. Endocrinology 2008, 149:5662-9.

54. Verdier Y, Penke B: Binding sites of amyloid beta-peptide in cell plasma membrane and implications for Alzheimer's disease. Curr Protein Pept Sci 2004, 5:19-31.

55. Goto Y, Kakizaki M, Masaki N: Production of spontaneous diabetic rats by repetition of selective breeding. Tohoku J Exp Med 1976, 119:85-90.

56. Livak KJ, Schmittgen TD: Analysis of relative gene expression data using real-time quantitative PCR and the 2(-Delta Delta $C(T))$ Method. Methods 2001, 25:402-8.

doi:10.1186/1471-2164-13-81

Cite this article as: Abdul-Rahman et al:: Altered gene expression profiles in the hippocampus and prefrontal cortex of type 2 diabetic rats. BMC Genomics 2012 13:81.

\section{Submit your next manuscript to BioMed Central and take full advantage of:}

- Convenient online submission

- Thorough peer review

- No space constraints or color figure charges

- Immediate publication on acceptance

- Inclusion in PubMed, CAS, Scopus and Google Scholar

- Research which is freely available for redistribution

Submit your manuscript at www.biomedcentral.com/submit
C BioMed Central 